\title{
A superconducting CW-LINAC for heavy ion acceleration at GSI
}

\author{
Winfried Barth ${ }^{1,2,5^{*}}$, Kurt Aulenbacher ${ }^{2,4}$, Markus Basten ${ }^{3}$, Florian Dziuba ${ }^{2}$, Viktor \\ Gettmann $^{2}$, Maksym Miski-Oglu ${ }^{2}$, Holger Podlech ${ }^{3}$ and Stepan Yaramyshev ${ }^{1,5}$ \\ ${ }^{1}$ GSI Helmholtzzentrum, 64291 Darmstadt, Germany \\ ${ }^{2} \mathrm{HIM}$, Helmholtzinstitut, 55128 Mainz, Germany \\ ${ }^{3}$ IAP, Goethe University, 60438 Frankfurt, Germany \\ ${ }^{4} \mathrm{KPH}$, Johannes Gutenberg-University, 55099 Mainz, Germany \\ ${ }^{5} \mathrm{MEPhI}$, National Research Nuclear University - Moscow Engineering Physics Institute, 115409 \\ Moscow, Russia
}

\begin{abstract}
Recently the Universal Linear Accelerator (UNILAC) serves as a powerful high duty factor $(25 \%)$ heavy ion beam accelerator for the ambitious experiment program at GSI. Beam time availability for SHE (Super Heavy Element)-research will be decreased due to the limitation of the UNILAC providing Uranium beams with an extremely high peak current for FAIR simultaneously. To keep the GSI-SHE program competitive on a high level and even beyond, a standalone superconducting continuous wave $(100 \%$ duty factor $)$ LINAC in combination with the upgraded GSI High Charge State injector is envisaged. In preparation for this, the first LINAC section (financed by HIM and GSI) will be tested with beam in 2017, demonstrating the future experimental capabilities. Further on the construction of an extended cryo module comprising two shorter Crossbar-H cavities is foreseen to test until end of 2017. As a final R\&D step towards an entire LINAC three advanced cryo modules, each comprising two $\mathrm{CH}$ cavities, should be built until 2019, serving for first user experiments at the Coulomb barrier.
\end{abstract}

\section{Introduction}

Design and construction of $\mathrm{cw}$ high intensity proton and ion linacs is a crucial goal of worldwide accelerator technology development [1-4]. Especially compactness of an accelerator facility is a modern trend, which high intensity $\mathrm{cw}$ proton and ion linac development has to comply with [5-7]. In the low and medium-energy range cw-linacs can be used for several applications, as boron-neutron capture therapy (BNCT), high productivity isotope generation and material science. The high energy linac is an integrated and essential part of several large scale research facilities, as spallation neutron sources or accelerator driven systems. Therefore the study and investigation of the design, operation and optimization of cw-linacs, as well as progress in elaboration of the superconducting technology, is of superior interest for the accelerator community around the world.

\footnotetext{
* Corresponding author: w.barth@gsi.de
} 
Providing heavy ion beams for the ambitious experiment program at GSI, the Universal Linear Accelerator (UNILAC) combined with the High Charge State Injector (HLI) served at last as a powerful high duty factor $(25 \%)$ accelerator. An UNILAC upgrade program is ongoing, designated to prepare for high intensity high current synchrotron injector operation for FAIR (Facility for Antiproton and Ion Research) [8-10]. As a result beam time availability for SHE-research will be strongly diminished due to the duty factor limitation for the UNILAC FAIR injector operation. To keep the SHE program at GSI competitive [11], an upgrade program of the HLI was already initialized comprising a new $28 \mathrm{GHz}$ ECR source and a cw capable RFQ and an IH-DTL [12]. A standalone sc cwLINAC [13] in combination with the upgraded HLI is assumed to meet the demands of the experimental program at its best. With significantly higher beam intensity the SHE production rate will be increased as well.

\section{General cw-LINAC layout}

A conceptual layout [13] of a sc cw-LINAC was worked out, which allows the acceleration of highly charged ions with a mass to charge ratio of 6 at $1.4 \mathrm{MeV} / \mathrm{u}$ from the upgraded HLI. Nine superconducting $\mathrm{CH}$ cavities operated at $217 \mathrm{MHz}$ accelerate the ions to energies between $3.5 \mathrm{MeV} / \mathrm{u}$ and $7.3 \mathrm{MeV} / \mathrm{u}$, while the energy spread should be kept smaller than $\pm 3 \mathrm{keV} / \mathrm{u}$. Superconducting solenoids mounted between the cavities provide for proper beam focusing. The general parameters are listed in table 1 .

Table 1. Design parameters of the cw-LINAC.

\begin{tabular}{|l|l|l|}
\hline Mass/Charge & & 6 \\
\hline Frequency & $\mathrm{MHz}$ & 217 \\
\hline Max. beam current & $\mathrm{mA}$ & 1 \\
\hline Injection Energy & $\mathrm{AMeV}$ & 1.4 \\
\hline Output energy & $\mathrm{AMeV}$ & $3.5-7.3$ \\
\hline Output energy spread & $\mathrm{AkeV}$ & \pm 3 \\
\hline Length of acceleration & $\mathrm{m}$ & 12.7 \\
\hline Sc CH-cavities & & 9 \\
\hline Sc solenoids & & 7 \\
\hline
\end{tabular}

$R \& D$ and prototyping (demonstrator project) $[14,15]$ in preparation of the proposed cw-LINAC is assigned to a collaboration of GSI, IAP and HIM, which was founded in 2009. The selected location to setup the demonstrator is in straightforward direction of the HLI at GSI (figure 1).

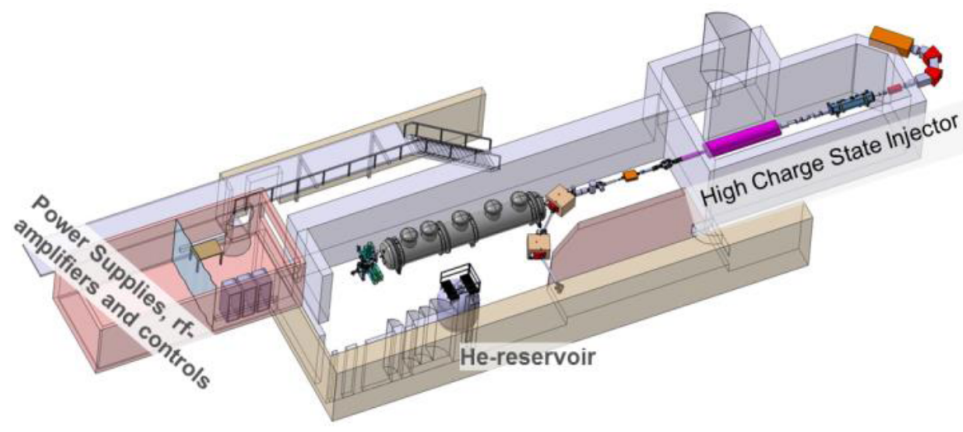

Figure 1.CH-multi cavity test environment@GSI. 
Two existing experiments at the HLI had to move since the space is needed for the demonstrator test environment embedded in a new radiation protection cave. The liquid helium (LHe) supply is covered by a 30001 tank. The consumed helium is collected in a $25 \mathrm{~m} 3$ recovery balloon and bottled by a compressor. For matching the beam from the HLI to the demonstrator a new rebuncher cavity as well as an existing rebuncher and an additional quadrupole doublet will be used. Moreover beam diagnostic devices, like SEMprofile grids and an emittance measurement devices and phase probes for beam energy measurements applying time of flight (TOF) has to be integrated in the beam line in front of and behind the demonstrator as well.

\section{Status of the UNILAC High Current Performance}

The GSI-UNILAC (figure 2) as well as the heavy ion synchrotron SIS18 will serve as a high current heavy ion injector for the new FAIR synchrotron SIS100.

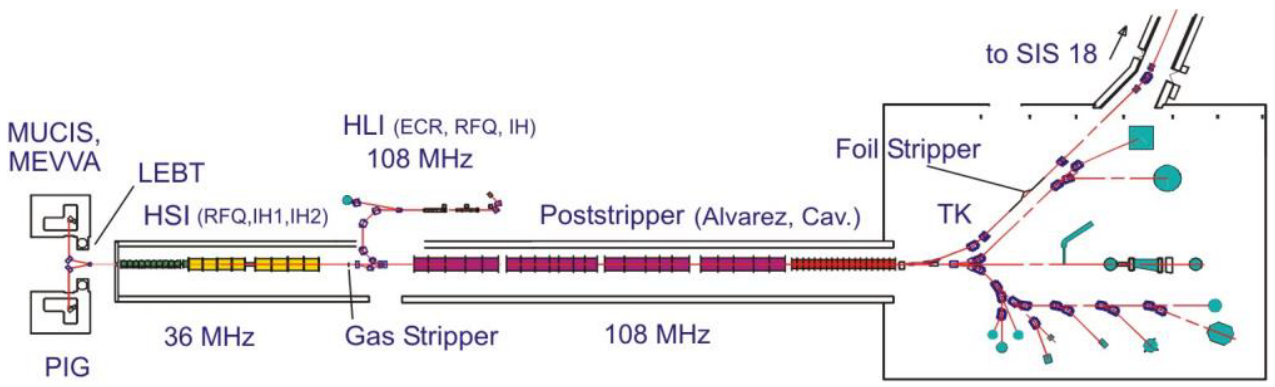

Figure 2. Layout of GSI UNILAC.

In the context of an advanced machine investigation program [16-18] in combination with the ongoing UNILAC upgrade program, a new uranium beam intensity record (11.5 emA, $\mathrm{U}^{29+}$ ) at very high beam brilliance was achieved recently in a machine experiment campaign. This is an important step paving the way to fulfill the FAIR heavy ion high intensity beam requirements. The $\mathrm{U}^{4+}$-beam current and brilliance was improved by applying a 7 hole-aperture extraction system at the VARIS ion source [19]. By optimization of the low energy beam transport and improved RFQ matching an RFQ transmission of $75 \%$ (11.3 emA) was achieved.

After advanced RF optimization and RF conditioning the HSI RFQ tank yields for reliable high-current uranium beam operation. Optimizing the MEBT (Medium Energy Beam Transport) between RFQ and IH DTL by increasing the transverse and longitudinal focusing the previously disturbing beam losses could be minimized significantly, resulting in a stable overall high current operation. Beam matching to the gas stripper by adapting the quadrupole channel resulted in a beam transmission of $90 \%$ in this section. For the first time an $\mathrm{U}^{4+}$ beam current of $7.6 \mathrm{emA}$ was available for heavy ion stripping. Applying a $\mathrm{H}_{2-}$ stripper gas cell at an optimal target thickness $\left(\approx 14 \mu \mathrm{g} / \mathrm{cm}^{2}\right)$ and after re-optimization of the charge separation procedure under high current conditions, an increased stripping efficiency of about $21 \%$ was achieved. Finally the uranium beam intensity at injection into the poststripper accelerator was increased by almost a factor of 4 accomplished in a 22 months lasting machine optimizing program (figure 3 ). 


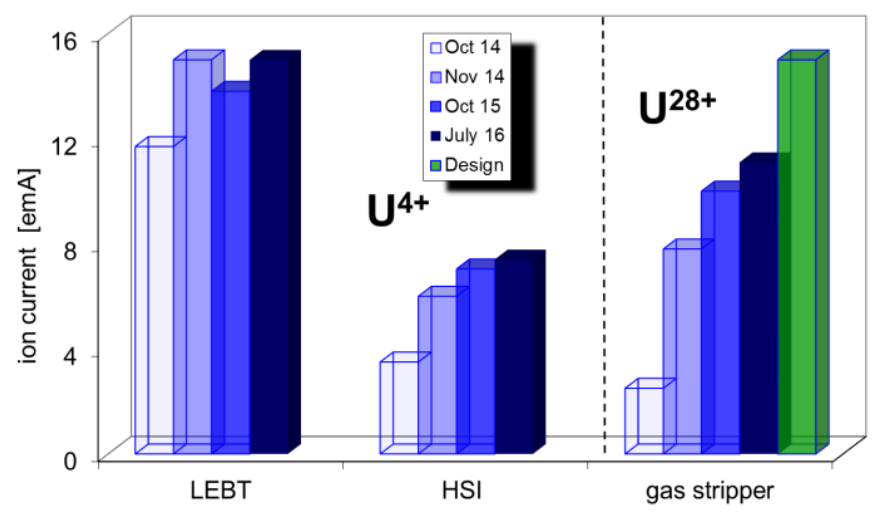

Figure 3. Measured uranium beam transmission at GSI-HSI and stripper section.

For this new record Uranium beam intensity $\left(11.5 \mathrm{~mA}, \mathrm{U}^{29+}\right)$ the beam emittance was measured carefully: The vertical beam emittance is significantly increased, while the horizontal emittance is decreased at higher beam current. For the high current beam dynamics layout of the gas stripper section, an enlarged vertical beam envelope in the interaction zone is foreseen, resulting also in an enhanced beam emittance growth due to strong particle straggling. Regarding high space charge power at higher ion beam current, the horizontal beam spot at the stripper has to be reduced as well. Thus horizontal beam brilliance at $1.4 \mathrm{MeV} / \mathrm{u}$ simply scales with the pulse current [9].

\section{Status of cw-LINAC}

The demonstrator (figure 4) [20] is the prototype of the first section of the proposed cwLINAC, comprising a superconducting $\mathrm{CH}$ cavity embedded by two superconducting solenoids. A study was conducted, which showed an assembly concept for the cryostat comprising the solenoids and the cavity as well. It is intended to align the three components to the beam axis. The sc $\mathrm{CH}$ structure is the key component and offers a variety of research and development. The beam focusing solenoids provide maximum fields of $9.3 \mathrm{~T}$ at an overall length of $380 \mathrm{~mm}$ and a free beam aperture of $30 \mathrm{~mm}$.

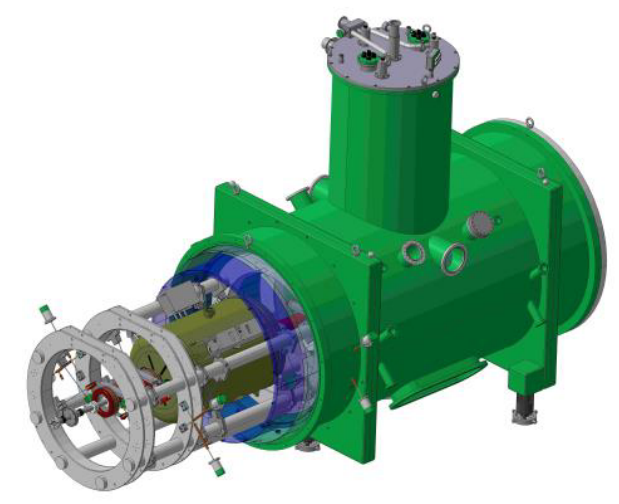

Figure 4. Cryostat with $\mathrm{CH}-$ cavity, high field solenoids, cold warm transitions and support system (Cryogenic Limited). 
The demonstrator project kick-off took place in 2010. After complete fabrication all acceptance tests passed through, and all subcomponents are delivered. For the design of the support frame, which carries the cavity (embedded by the two sc solenoids), a suspension concept was applied (figure 5) [21, 22]. The support frame, as well the accelerator components, are suspended each by eight tie rods in a cross-like configuration balancing the mechanical stress during the cooldown and warm up (figure 6). As a consequence the suspended components may always stay within the tolerance limits related to the beam axis. Another important feature concerns the two sc solenoids: A configuration of one main coil out of $\mathrm{NbSn}$ and two compensation coils made from $\mathrm{NbTi}$ shields the maximum magnetic field of $9.3 \mathrm{~T}$ within a longitudinal distance of $10 \mathrm{~cm}$ down to $30 \mathrm{mT}$, acceptable at the position of the neighbored cavity (first RF-gap). The solenoids are connected to LHe ports inside the cryostat by copper tapes allowing dry cooling.

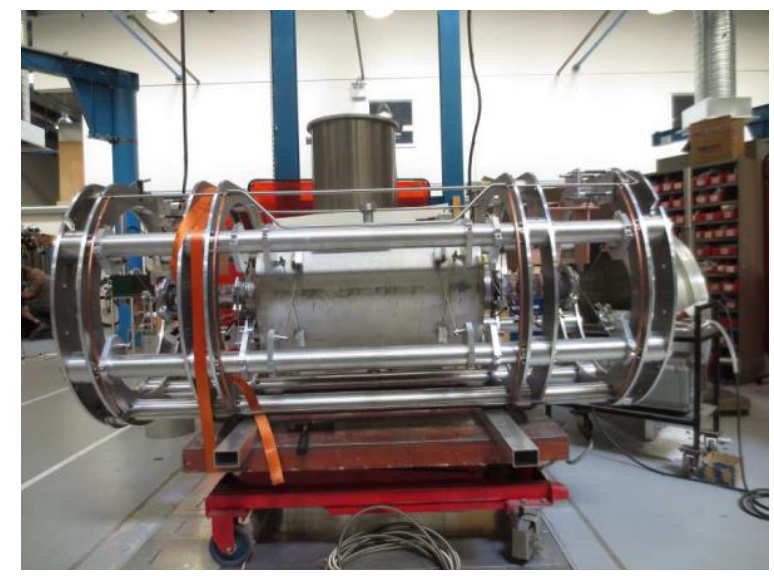

Figure 5. Sc cw-LINAC-demonstrator comprising a $\mathrm{CH}$ cavity embedded by two sc solenoids on a support frame, to be mounted inside the cryostat.

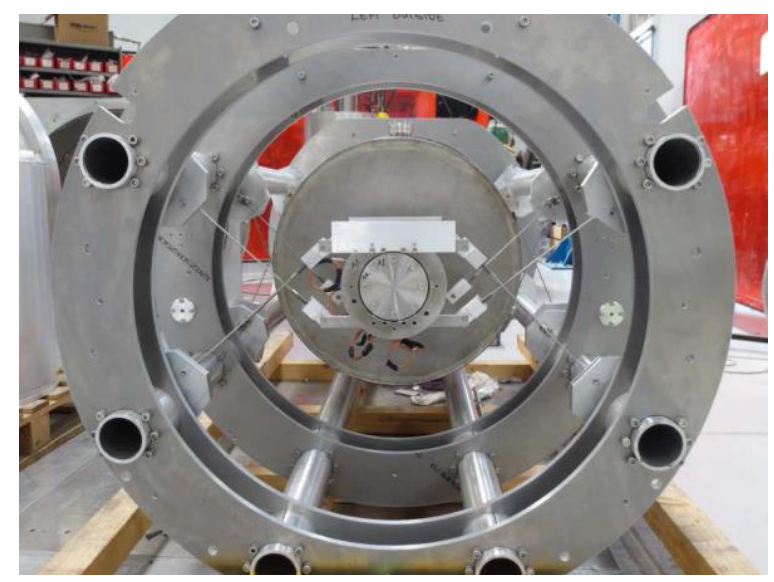

Figure 6. Detailed view of tie rods in a cross-like configuration.

The CH cavity is directly cooled with LHe supported by a helium jacket out of titanium. The vendor RI (Research Instruments GmbH, Germany) provided for sufficient cavity preparation. After final high pressure rinsing (HPR), the delivery of the cavity was accomplished in September 2015; a performance test at low temperature (4.2 K) and with 
low RF power was performed in October 2015 at Goethe University Frankfurt [23]. Three piezo frequency tuners are manufactured at GSI, tuning the resonance frequency, while RFoperation. Therefore a tuner dummy was already tested at Goethe University Frankfurt [24]. Commissioning of the demonstrator is planned in 2016 at the GSI HLI, which operates at $108 \mathrm{MHz}$ (Figure 1). A new beam line in straightforward direction to the HLI, which transports the beam to the new radiation protection shelter locating the demonstrator, was designed regarding beam dynamical simulations. In June 2015 an emittance measurement at the future position of the demonstrator cryostat confirmed the latest beam dynamic simulations and the aspired positioning of the components in the beam transport line [25].

\section{Advanced R\&D}

The successor of the demonstrator R\&D is already the "Advanced Demonstrator" project. It is planned to build the first half of the future entire cw LINAC. Based on calculations of the beam dynamics [26, 27], the cw LINAC cryo module, comprising two $\mathrm{CH}$ cavities and one superconducting solenoid, was defined.

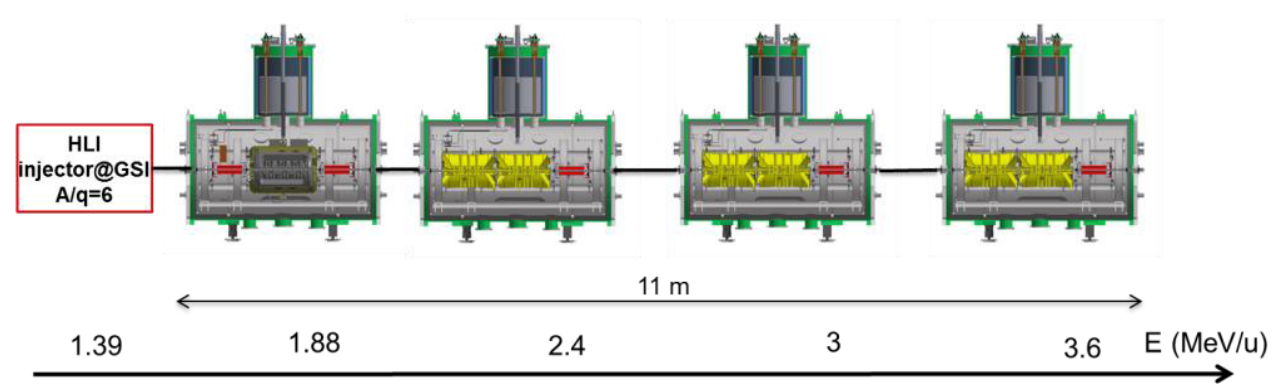

Figure 7. Layout of the Advanced Demonstrator.

Figure 7 shows the present layout based on the so called "short cavity" design. The usage of short $\mathrm{CH}$ cavities allows more flexibility in tuning the entire cw LINAC, maintaining the necessity of variable output energy and almost a neglect able emittance growth during the acceleration process. The design value of the accelerating gradient of $\mathrm{E}_{\mathrm{a}}=5.5 \mathrm{MV} / \mathrm{m}$ provides an effective accelerating voltage of $1.8 \mathrm{MV}$ per (short) cavity. A sc solenoid with a maximum magnetic field of $9.3 \mathrm{~T}$ is sufficient to focus the heavy ion beam in both transversal planes. A cryo plant with a total power of $2.3 \mathrm{~kW}$, currently used for the testing of superconducting dipole magnets for FAIR-SIS100, is foreseen to supply the Advanced Demonstrator by two cooling loops $\left(4.4^{\circ} \mathrm{K}\right.$ at 3.5 bar and $50^{\circ} \mathrm{K}$ at 11 bar respectively). The cryogenic power is delivered to the local helium distribution system via a double shielded transfer line. Each cryo module has its own distribution box with a JouleThompson expansion valve, producing liquid helium; a controlled valve maintains a constant pressure in the cavities within 10 mbar. The cryostat provides for the $4.2 \mathrm{~K}$ environment for solenoid as well as for the cavities with static losses of maximum $5 \mathrm{~W}$. The accelerator components are mounted on a support frame by a so called Nuclotron suspension, which allows preserving the alignment of components during the cooldown within $0.2 \mathrm{~mm}$ (in transverse direction). The support frame itself is also suspended by a Nuclotron suspension, allowing for adjustment of the whole frame in situ under operational conditions. 


\section{Summary \& Outlook}

A UNILAC upgrade program is successfully ongoing, designated to prepare for high intensity high current synchrotron injector operation for FAIR. As a result beam time availability for SHE-research will be strongly diminished. A standalone sc cw-LINAC is envisaged to keep the GSI-SHE program competitive on a high level and even beyond. In preparation for this, the first LINAC section is already built as a demonstrator. After the successful RF-test of the demonstrator cavity further activities in preparation of the first beam test are conducted: Cavity treatments, pushing the accelerating gradient to the theoretical limit of about $25 \mathrm{MV} / \mathrm{m}$, as well as ordering of components for the advanced demonstrator project. The beam test of the demonstrator cavity is scheduled for March 2017.

\section{References}

1. C. Prior, Overview of High Intensity Accelerator Projects, Proceedings of HB'10 (Morschach, Switzerland, 2010)

2. T.V. Kulevoy, S.M. Polozov, A.D. Fertman et al., Atomic Energy 117(5), 117:347 (2015)

3. P.N. Ostroumov, Advances in CW Ion Linacs, Proceedings of IPAC'15 (Richmond, VA, USA, 2015)

4. N. Solyak et al., The Concept Design of the CW Linac of the Project X, Proceedings of IPAC'10 (Kyoto, Japan, 2010)

5. S.M. Polozov et al., Beam Dynamics Simulations and Code Comparison for a New CW RFQ Design, Proceedings of HB'16 (Malmö, Sweden, 2016)

6. L. Weissman, D. Berkovits et al., Journal of Instrumentation 10, T1004 (2015)

7. G.V. Trubnikov et al., Physics of Particles and Nuclei Letters 13(7), 915-918 (2016)

8. W. Barth et al., Phys. Rev. ST Accel. Beams 18(4), 040101 (2015)

9. W. Barth et al., High Current Uranium Beam Measurements at GSI-UNILAC for FAIR, Proceedings of HB'16 (Malmö, Sweden, 2016)

10. W. Barth et al., Phys. Rev. ST Accel. Beams 18(5), 050102 (2015)

11. S. Hofmann et al., Heavy Element Research at GSI-SHIP, Proceedings of EXON'04 (Peterhof, Russia, 2004)

12. W. Barth et al., UNILAC-upgrade programme for the heavy element research at GSISHIP, Proceedings of EPAC'06 (Edinburgh, Scotland, 2006)

13. S. Minaev et al., Phys. Rev. ST Accel. Beams 12, 120101 (2009)

14. S. Mickat et al., The sc cw-LINAC Demonstrator - SRF technology finds the way to GSI, Proceedings of SRF'11 (Chicago, USA, 2011)

15. V. Gettmann et al., The sc cw-LINAC Demonstrator - an R\&D project for SRF technology, Proceedings of SRF'11 (Chicago, USA, 2011)

16. W. Barth et al., Upgrade program of the high current heavy ion UNILAC as an injector for FAIR, NIM A 577(1-2), 211-214 (2007)

17. S. Yaramyshev et al., Phys. Rev. ST Accel. Beams 18(4), 050103 (2015)

18. S. Yaramyshev et al., Development of the versatile multi particle code DYNAMIION, NIM A 558(1), 90-94 (2006)

19. R. Hollinger et al., Nucl. Instrum. Meth. B 239, 227 (2005)

20. S. Mickat, M. Amberg, K. Aulenbacher, W. Barth, F. Dziuba, V. Gettmann, S. Jacke, A. Orzhekhovsaya, H. Podlech, U. Ratzinger, K. Tinschert, Status of the superconducting cw LINAC at GSI. DOI: 10.1142/9789814508865_0044 (Vladivostok, Russia, 2013) 
21. V. Gettmann et al., The sc cw -LINAC Demonstrator - First section of a sc cw LINAC, Proceedings of SRF'11 (Chicago, Illinois, USA, 2011)

22. V. Gettmann et al., Recent status new superconducting cw heavy ion LINAC@GSI, Proceedings of SRF'15 (Whistler, Canada, 2015)

23. F. Dziuba et al., First Performance Test on the Superconducting $217 \mathrm{MHz} \mathrm{CH}$ Cavity at $4.2 \mathrm{~K}$, Proceedings of LINAC'16 (East Lansing, MI, U.S.A., 2016)

24. M. Amberg et al., The fast Piezo-based frequency tuner for sc CH-cavities, Proceedings of LINAC'14 (Geneva, Switzerland, 2014)

25. M. Miski-Oglu, et al., Steps towards superconducting cw-LINAC for heavy ions at GSI, Proceedings of SRF'15 (Whistler, Canada, 2015)

26. M. Schwarz et al., Beam Dynamics for the sc $\mathrm{cw}$ heavy ion LINAC at GSI, Proceedings of IPAC'15 (Richmond, U.S.A., 2015)

27. S. Yaramyshev et al., An Advanced Procedure for Longitudinal Beam Matching for SC CW Heavy Ion LINAC With Variable Output Energy, Proceedings of HB'16 (Malmö, Sweden, 2016) 